\title{
Primary headaches interfere with the efficacy of temporomandibular disorders management
}

\author{
André Luís PORPORATTI ${ }^{1}$, Yuri Martins COSTA ${ }^{1}$, Paulo César Rodrigues CONTI', Leonardo Rigoldi BONJARDIM², \\ Patrícia dos Santos CALDERON ${ }^{3}$ \\ 1- Department of Prosthodontics, Bauru School of Dentistry, University of São Paulo, Bauru, SP, Brazil. \\ 2- Department of Biological Sciences, Bauru School of Dentistry, University of São Paulo, Bauru, SP, Brazil. \\ 3- Department of Dentistry, Federal University of Rio Grande do Norte, Natal, RN, Brazil. \\ Corresponding address: André Luís Porporatti - Departamento de Prótese - Faculdade de Odontologia de Bauru - Universidade de São Paulo - Al. Octávio \\ Pinheiro Brizolla, 9-75 - Vila Universitária- 17012-901 - Bauru - SP - Brazil \\ e-mail: andreporporatti@yahoo.com.br / andreporporatti@usp.br
}

Submitted: October 29, 2013 - Modification: February 10, 2014 - Accepted: March 31, 2014

\section{ABSTRACT}

\begin{abstract}
bjectives: This cross-sectional study aimed to evaluate the influence of Primary Headache (PH) on efficacy of a Temporomandibular Disorders (TMD) conservative therapy and its association with the presence of self-reported parafunctional habits. Sample and Methods: Sample was composed of 400 medical records, divided into four groups: I) Muscular TMD ( $n=64)$; II) Muscular TMD+PH $(n=48)$; III) Muscular TMD+Articular TMD $(n=173) ; I V)$ Muscular TMD+Articular TMD+PH $(n=115)$. All groups had undergone a TMD therapy for three months with a stabilization appliance and counseling for habits and behavioral changes, with no specific headache management. Current pain intensity and existence or not of self-reported bruxism were assessed. Repeated measures ANOVA and Chi-Square test followed by Odds were used for statistical analysis, with a significance level of $5 \%$. Results: results of this study showed that: (1) A conservative therapy with stabilization appliance and counseling for habits and behavioral changes was effective in the TMD pain relief; (2) Groups with an additional diagnosis of PH had worsened the pain improvement significantly; and (3) no association between the presence of self-reported bruxism and PH was found. Conclusions: this study could elucidate the important effect that headache may have on the TMD management.
\end{abstract}

Keywords: Facial pain. Temporomandibular joint disorders. Headache. Therapy.

\section{INTRODUCTION}

Primary headache $(\mathrm{PH})$ and chronic orofacial pain are common symptoms in the general population ${ }^{8}$, and the presence of headache in patients reporting temporomandibular disorders (TMD) have a high prevalence of 50 to $80 \%{ }^{6,8,28}$, whereas these numbers in patients without TMD are only 13 to $23 \%^{19,33}$.

Primary headache individuals could experience more severe and frequent symptoms of TMD, and vice-versa ${ }^{2,3}$. The relationship between these two entities, however, is not well established in the literature yet $t^{5,19}$.

Some studies have shown that TMD therapy reduces the complaint of headache in both intensity and frequency ${ }^{30}$, nevertheless, to the best of our knowledge, the impact of PH on TMD therapy efficacy has not been published yet.

Furthermore, some studies have confirmed that the presence of parafunctional habits, i.e., activities of the masticatory muscles, as clenching and grinding, has an important role in TMD pain, whereas patients with sleep bruxism have an increased risk for the occurrence of myofascial pain ${ }^{15}$. This association with headache is not well defined ${ }^{29}$.

Based on the above, this study aimed to evaluate the influence of the presence of $\mathrm{PH}$ on the efficacy of a TMD conservative therapy and the association with the presence of self-reported parafunctional habits. The null hypothesis is that the presence of primary headaches does not influence negatively the efficacy of TMD management. 


\section{SAMPLE AND METHODS}

This study was approved by the Institutional Ethics Committee, under protocol \#105/2010.

\section{Sample}

This cross-sectional study performed 1,200 evaluations in medical records from an Orofacial Pain Clinic, from 1996 to 2009. The sample was composed of 400 medical records, initially selected according to the following criteria:

Inclusion:

1. Medical records of adults aged 18 years or more with:

2. Presence of myofascial pain with or without the presence of muscular trigger points by manual palpation, according to the American Academy of Orofacial Pain (AAOP) ${ }^{11}$;

Exclusion:

1. Medical records of individuals with neurological diseases, fibromyalgia, systemic arthritis, secondary headache or paroxysmal hemicrania, menstrual and cluster headache.

\section{Group establishment}

After fulfilling the initial criteria, the medical records were analyzed according to the presence or not of TMD through AAOP criteria ${ }^{11}$; and $\mathrm{PH}$ based on International Headache Society (IHS) ${ }^{24}$, fulfilling the criteria for migraine and/or tensiontype headache.

Patients could be allocated into one of the four groups below:

\section{I) Only muscular TMD (with no headache complaint):}

This group was composed of 64 medical records of subjects with muscular TMD only. Muscular TMD encompassed localized myalgia and masticatory myofascial pain according to AAOP ${ }^{11}$.

They had undergone a conservative therapy for three months with a rigid acrylic upper full coverage stabilization appliance, along with counseling for habits and behavioral changes, home care instructions, exercises and Physical therapy modalities $^{9}$. Analgesics and anti-inflammatory drugs could also be used.

\section{II) Muscular TMD+primary headache:}

This group was composed of 48 medical records of subjects with muscular TMD plus the presence of primary headache. They had undergone the same conservative therapy for group I, with no specific headache management.

III) OnIy muscular TMD+articular TMD (with no headache complaint):

This group was composed of 173 medical records from subjects with muscular and articular TMD. They had undergone the same conservative therapy for group I.

Articular TMD included arthralgia and disc displacement with or without reduction, with or without mouth opening limitation, fulfilling the AAOP criteria ${ }^{11}$.

\section{IV) Muscular TMD+articular TMD+primary headache:}

This group was composed of 115 medical records of subjects with muscular and articular TMD plus the presence of primary headache. They had undergone the same conservative therapy for group I, with no specific headache management.

\section{Examiners and variables}

Two examiners performed all the medical records evaluations. Current pain intensity was analyzed according to self-reported pain on Visual Analogue Scale (VAS) at baseline and after three months of TMD therapy. Moreover, the existence or not of selfreported clenching/bruxism parafunctional habits was analyzed at baseline. The pain experience duration, in months, was also recorded.

\section{Data analysis}

The results were analyzed with repeated measurements ANOVA (after Kolmogorov-Smirnov to test normal distribution) to assess within and between groups differences; Chi-Square test followed by Odds Ratio test, with $95 \%$ of Confidence Interval ( $\mathrm{CI}$ ), to test association between $\mathrm{PH}$ and bruxism; and Spearman Correlation to correlate pain duration and pain intensity variation between baseline and after therapy. The results were established with a significance level of $5 \%$.

\section{RESULTS}

The mean age was 36.87 years for group I, 36.79 years for group II, 36.36 for group III, and 36.79 for group IV $(p=0.55)$. Women were $81.25 \%$ of group I, $89.58 \%, 86.12 \%$, and $94.78 \%$ of group II, III, and IV, respectively.

In the within groups analysis, there was a significant reduction in the reported pain for all groups $(p<0.05)$ after three months of TMD therapy (Figure 1).

Pain improvement in VAS was $70.74 \%$ (SD 30.71 ) and $46.32 \%$ (SD 24.70) for group I and II, respectively, and $66.44 \%$ (SD 35.37) and $42.56 \%$ (SD 31.72) for group III and IV, respectively (Figure 1). Significant results revealed better pain improvement for group I compared to group II, and for group III, when compared to group IV $(p<0.05 \%)$ (Figure 1).

At baseline, significant differences for the self- 
report of clenching/bruxism were perceived only between groups I and II with III and IV, which showed that subjects with muscular TMD had at baseline a higher percentage of clenching/bruxism parafunctional habits than subjects with muscular and articular TMD, without considering the presence or not of $\mathrm{PH}$ (Table 1). No differences were found on the association between $\mathrm{PH}$ and bruxism (Table 2).
The mean duration of pain experience was 63.71 months (SD 62.37), 70.95 (SD 76.85), 58.91 (SD 71.00), and 67.92 (SD 84.38) for group I, II, III, and IV, respectively $(p=0.10)$. No significant correlation with the pain improvement was detected (Spearman $\mathrm{R}=0.036, \mathrm{p}=0.39$ ).

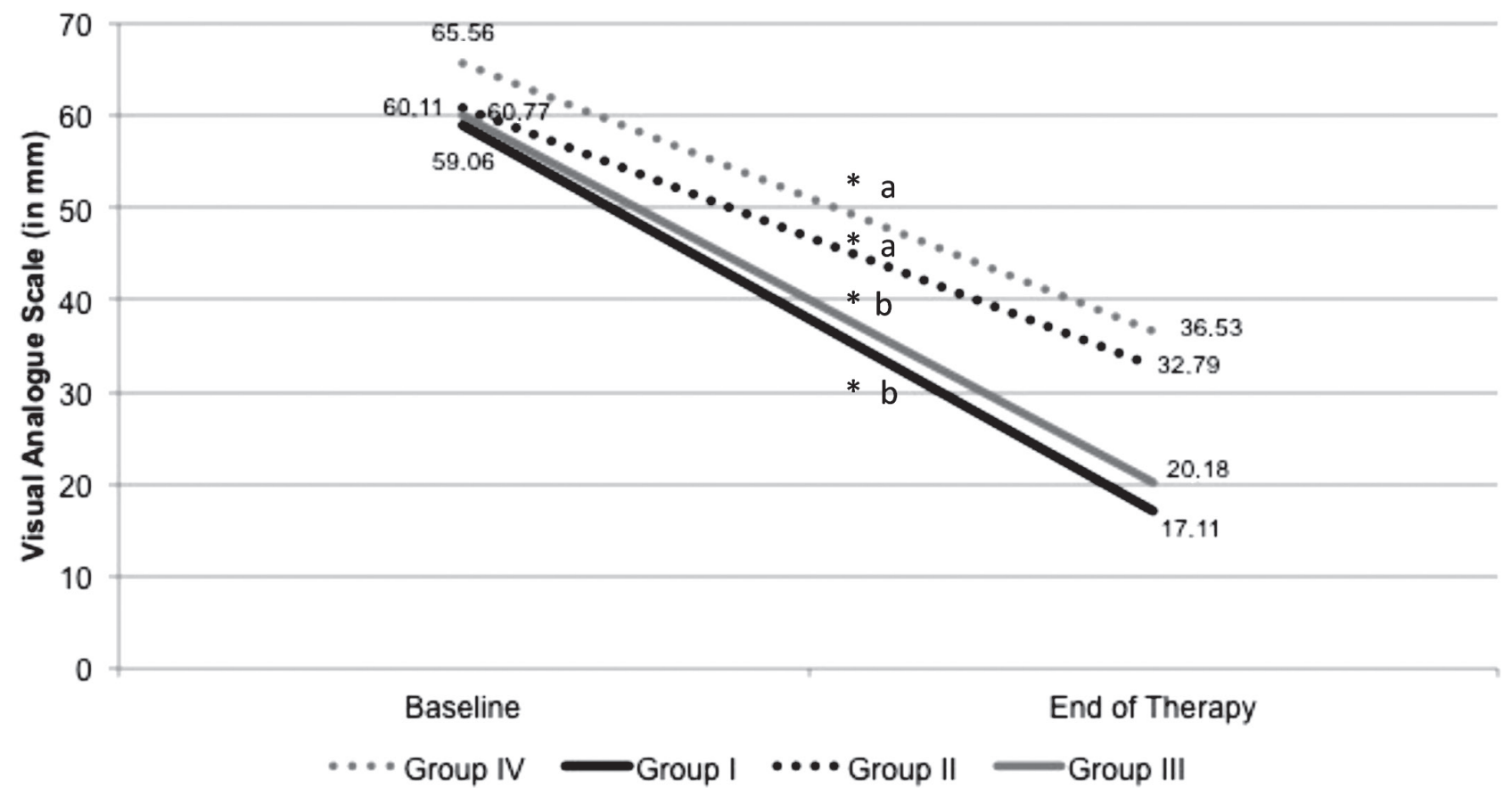

* indicates within-groups differences $(p<0.05)$

aa indicates no between-groups differences $(p>0.05)$

ab indicates between-groups differences $(p<0.05)$

Figure 1- Pain intensity according to Visual Analogue Scale (VAS) at baseline and in the end of the therapy

Table 1- Percentage of clenching/bruxism parafunctional habits between groups

\begin{tabular}{cccc}
\hline GROUP & TOTAL & PRESENCE OF CLENCHING/BRUXISM & PERCENTAGE \\
\hline I & 64 & 57 & $89.06 \%^{\mathrm{a}}$ \\
II & 48 & 42 & $87.50 \%^{\mathrm{a}}$ \\
III & 173 & 86 & $49.71 \%^{\mathrm{b}}$ \\
IV & 115 & 59 & $51.30 \%^{\mathrm{b}}$ \\
\hline
\end{tabular}

a indicates no between-groups differences $(p>0.05)$

$\mathrm{b}$ indicates between-groups differences $(p<0.05)$

Table 2- Chi-Square test (X2) and Odds Ratio (OR) of association between primary headache and clenching/bruxism parafunctional habits

\begin{tabular}{cccc}
\hline & HEADACHE & WITHOUT HEADACHE & TOTAL \\
\hline BRUXISM & $101(41.4 \%)$ & $143(58.6 \%)$ & 244 \\
WITHOUT BRUXISM & $62(39.7 \%)$ & $94(60.3 \%)$ & 156 \\
TOTAL & 163 & 237 & 400 \\
\hline
\end{tabular}

$\mathrm{p}=0.743, \mathrm{OR}=1.07(0.71-1.61)$ 


\section{DISCUSSION}

This study evaluated 400 medical records regarding the efficacy of TMD management in patients with primary headache as a comorbid condition. The null hypothesis was rejected. The main findings were: (1) a conservative therapy with stabilization appliance and counseling for habits and behavioral changes was effective in the TMD pain relief; (2) the presence of concomitant primary headache negatively influenced the efficacy of TMD therapy; (3) no association between the presence of self-reported bruxism and PH was found; and (4) no correlation was found between TMD management efficacy on pain severity and the duration of pain experience.

Our sample was mainly composed of women (88\%), aged between $30-45$ years (35.47 \pm 12.59$)$, which is in agreement with epidemiological publications ${ }^{17,27}$. All groups had chronic craniofacial pain, with mean duration of $63.72 \pm 74.30$ months.

After three months of therapy, management strategies as stabilization splint and counseling for behavioral changes used in the present study provided a significant improvement in the pain levels of articular and/or muscular TMD when judged by the VAS. This management approach is supported by the literature and is recommended as an effective therapy modality $7,16,17,21,22,32$.

A possible explanation on the use of this management approach is based on a decrease of the overload masticatory system ${ }^{2,23}$. Stabilization splints provide proper occlusal relationship, reduction in muscular activity, stable physiologic mandibular posture; and with the potential cognitive and placebo effect ${ }^{20,31}$. The actual results may have an essential correlation with all these mechanisms of action. Furthermore, the patient education about behavioral changes, the probable high impact of the patient's initial reaction to a professional orientation, and also placebo effect, natural evolution of the disease and regression towards the mean ${ }^{9}$ may play a role in the pain reduction for all groups observed in this study.

After the TMD management, groups with additional diagnosis of primary headache did not have the same level of amelioration than groups with only TMD and without PH. It has been very well established that nociceptive impulses arising from masticatory muscles and temporomandibular joint, as well as cranial tissues and head area, may induce central sensitization mechanisms in the subnucleus caudalis of the trigeminal nerve system ${ }^{19}$. Since both areas, TMD and head region, share similar ascendant pain pathways in the trigeminal system, the increase in pain transmission from peripheral tissues to the trigeminal system could explain the fact that the presence of comorbid $\mathrm{PH}$, not treated, could influence negatively a TMD therapy, and the approach of both conditions should be stated.

The effectiveness of stabilization devices in the management of TMD pain is well documented and it is related to the re-establishment of proper occlusal relationship; the reduction in muscular activity; the cognitive effect, since the patients became aware of their oral parafunctional habits; and the placebo effect $7,9,17,22,32$. However, the literature is vague about this fact in headache improvement. Some studies report headache pain decrease, in both intensity and frequency, about 30 to $50 \%$ when TMD was treated with splint therapy, in short and long-term evaluations $\mathbf{s}^{12,13,30}$.

The literature suggests that subjects with sleep bruxism have increased risk for myofascial and joint pain conditions ${ }^{15}$. In this study, the prevalence of clenching/bruxism parafunctional habits was very high for all groups.

Some studies had showed that the postexercise muscle soreness is associated with this overload mechanism of muscle pain in individuals with bruxism, which can be a risk factor to the installation of myofascial TMD $3,10,15$. Our results show higher prevalence of bruxism in the muscular TMD groups only, and lower values in the articular TMD groups. It is possible that this outcome could be a result of a difference in the pathophysiology between the articular and the muscular TMD. In the group presenting with articular and muscular $T M D$, the muscle pain might be a consequence of the articular pain conditions, which, in turn, could lead to a muscle co-contraction. In this case, the occurrence of parafunctional habits could not be as important as in the case of only muscle pain. Furthermore, the prevalence of bruxism in articular TMD patients is lower ${ }^{25}$. Finally, considering that muscle pain could be a symptom of bruxism ${ }^{4}$, in the group of only muscular TMD, bruxism could be the primary source of pain, which explains the higher prevalence in the groups I and II.

The causal relationship between bruxism and TMD is controversial. A recent systematic review found that studies based on self-report or clinical diagnosis of bruxism have a positive association with TMD, while studies based on more specific quantitative diagnostic methods revealed a weak association with symptoms of $\mathrm{TMD}^{26}$. Although parafunctional habits increase the activation of masticatory muscles ${ }^{18}$, which sometimes may cause pain ${ }^{1}$, bruxism is not always correlated with the pain caused by TMD. In this sense, to establish a direct causal relationship between bruxism and TMD is difficult.

Our study showed that self-reported bruxism was not associated with the presence of $\mathrm{PH}$. A recent study evaluated the association between TMD, sleep bruxism (SB) and PH. It could be found 
that in patients with painful TMD and SB, only the presence of sleep bruxism did not increase the risk for any kind of $\mathrm{PH}^{14}$.

No correlation between TMD improvement after three months and duration of pain experience was found. It was expected that the longer the pain experience, the higher the peripheral and central sensitization, associated to some sort of impairment of the descending inhibitory analgesic system, which could influence the efficacy of pain management. However, this fact was not tested here. The non-administration of central acting medication and strategies devoted to revert such central alterations could explain this similarity between those with more time of pain experience and those with less time.

Another limitation of the present study is the relatively weak reliability of the data obtained from the medical records, usually handled by different professionals. Although extracted from records from the same treatment center, variations in diagnosis and management strategies are expected, jeopardizing the present results.

Based on the aforementioned, this study showed the potential and significant effect that headache has in the efficacy of TMD management. A combined approach to both pain conditions is suggested.

\section{DISCLOSURE}

The authors declare that they have no conflicts of interest.

\section{REFERENCES}

1- Ahlberg K, Ahlberg J, Könönen M, Alakuijala A, Partinen M, Savolainen A. Perceived orofacial pain and its associations with reported bruxism and insomnia symptoms in media personnel with or without irregular shift work. Acta Odontol Scand. 2005;63(4):213-7.

2- Alencar F Jr, Becker A. Evaluation of different occlusal splints and counselling in the management of myofascial pain dysfunction. J Oral Rehabil. 2009;36(2):79-85.

3- Arima T, Svensson P, Arendt-Nielsen L. Experimental grinding in healthy subjects: a model for postexercise jaw muscle soreness? J Orofac Pain. 1999;13(2):104-14.

4- Bader G, Lavigne G. Sleep bruxism; an overview of an oromandibular sleep movement disorder. REVIEW ARTICLE. Sleep Med Rev. 2000;4(1):27-43.

5- Ballegaard V, Thede-Schmidt-Hansen P, Svensson P, Jensen R. Are headache and temporomandibular disorders related? A blinded study. Cephalalgia. 2008;28(8):832-41.

6- Bendtsen L, Jensen R. Tension-type headache. Neurol Clin. 2009;27(2):525-35.

7- Bergstrom I, List T, Magnusson T. A follow-up study of subjective symptoms of temporomandibular disorders in patients who received acupuncture and/or interocclusal appliance therapy 18-20 years earlier. Acta Odontol Scand. 2008;66(2):88-92.

8- Ciancaglini R, Radaelli G. The relationship between headache and symptoms of temporomandibular disorder in the general population. J Dent. $2001 ; 29(2): 93-8$.
9- Conti PC, Alencar EN, Mota Corrêa AS, Lauris JR, Porporatti AL, Costa YM. Behavioural changes and occlusal splints are effective in the management of masticatory myofascial pain: a short-term evaluation. J Oral Rehabil. 2012;39(10):754-60.

10- Dao TT, Lund JP, Lavigne GJ. Comparison of pain and quality of life in bruxers and patients with myofascial pain of the masticatory muscles. J Orofac Pain. 1994;8(4):350-6.

11- De Leeuw R, Klasser GD (editors). Orofacial pain: guidelines for assessment, diagnosis, and management. Chicago: Quintessence; 2008.

12- Doepel M, Nilner M, Ekberg E, Vahlberg T, Bell YI. Headache: short- and long-term effectiveness of a prefabricated appliance compared to a stabilization appliance. Acta Odontol Scand. 2011;69(3):129-36.

13- Ekberg EC, Nilner M. Treatment outcome of short- and longterm appliance therapy in patients with TMD of myogenous origin and tension-type headache. J Oral Rehabil. 2006;33(10):713-21. 14- Fernandes G, Franco AL, Goncalves DA, Speciali JG, Bigal ME, Camparis CM. Temporomandibular disorders, sleep bruxism, and primary headaches are mutually associated. J Orofac Pain. 2013;27(1):14-20.

15- Fernandes G, Franco AL, Siqueira JT, Goncalves DA, Camparis $\mathrm{CM}$. Sleep bruxism increases the risk for painful temporomandibular disorder, depression and non-specific physical symptoms. J Oral Rehabil. 2012;39(7):538-44.

16- Freitas RF, Ferreira MA, Barbosa GA, Calderon PS. Counselling and self-management therapies for temporomandibular disorders: a systematic review. J Oral Rehabil. 2013;40(11):864-74.

17- Fricton J. Myogenous temporomandibular disorders: diagnostic and management considerations. Dent Clin North Am. 2007;51(1):61-83.

18- Glaros AG, Burton E. Parafunctional clenching, pain, and effort in temporomandibular disorders. J Behav Med. 2004;27(1):91100.

19- Gonçalves DA, Speciali JG, Jales LC, Camparis CM, Bigal ME. Temporomandibular symptoms, migraine, and chronic daily headaches in the population. Neurology. 2009;73(8):645-6.

20- Greene CS, Goddard G, Macaluso GM, Mauro G. Topical review: placebo responses and therapeutic responses. How are they related? J Orofac Pain. 2009;23(2):93-107.

21- Helkimo M. Request for expert statement regarding the use of NTI-splint. Stockholm: Socialstyrelesen; 2003.

22- Klasser GD, Greene CS. Oral appliances in the management of temporomandibular disorders. Oral Surg Oral Med Oral Pathol Oral Radiol Endod. 2009;107(2):212-23.

23- Kopp S. Short term evaluation of counselling and occlusal adjustment in patients with mandibular dysfunction involving the temporomandibular joint. J Oral Rehabil. 1979;6(2):101-9.

24- Lipton RB, Bigal ME, Steiner TJ, Silberstein SD, Olesen J. Classification of primary headaches. Neurology. 2004;63(3):427435.

25- Manfredini D, Cantini E, Romagnoli M, Bosco M. Prevalence of bruxism in patients with different research diagnostic criteria for temporomandibular disorders (RDC/TMD) diagnoses. Cranio. $2003 ; 21(4): 279-85$

26- Manfredini D, Lobbezoo F. Relationship between bruxism and temporomandibular disorders: a systematic review of literature from 1998 to 2008. Oral Surg Oral Med Oral Pathol Oral Radiol Endod. 2010;109(6):e26-50.

27- Manfredini D, Piccotti F, Ferronato G, Guarda-Nardini L. Age peaks of different RDC/TMD diagnoses in a patient population. J Dent. 2010;38(5):392-9.

28- Mitrirattanakul S, Merrill RL. Headache impact in patients with orofacial pain. J Am Dent Assoc. 2006;137(9):1267-74.

29- Nagamatsu-Sakaguchi C, Minakuchi H, Clark GT, Kuboki T. Relationship between the frequency of sleep bruxism and the prevalence of signs and symptoms of temporomandibular disorders in an adolescent population. Int J Prosthodont. 2008;21(4):292-8. 
30- Nilsson $\mathrm{H}$, Vallon D, Ekberg EC. Long-term efficacy of resilient appliance therapy in TMD pain patients: a randomised, controlled trial. J Oral Rehabil. 2011;38(10):713-21.

31- Pollo A, Carlino E, Benedetti F. Placebo mechanisms across different conditions: from the clinical setting to physical performance. Philos Trans R Soc Lond B Biol Sci. 2011;366(1572):1790-8.
32- Stapelmann H, Türp JC. The NTI-tss device for the therapy of bruxism, temporomandibular disorders, and headache - where do we stand? A qualitative systematic review of the literature. BMC Oral Health. 2008;8:22.

33- Wiendels NJ, Knuistingh Neven A, Rosendaal FR, Spinhoven P, Zitman FG, Assendelft WJ, et al. Chronic frequent headache in the general population: prevalence and associated factors. Cephalalgia. 2006;26(12):1434-42. 